\title{
Quantitative Real-Time Imaging of Glutathione with Subcellular Resolution
}

\author{
Xiqian Jiang, ${ }^{1, *}$ Chengwei Zhang,,,* Jianwei Chen,, ${ }^{1, *}$ Sungwoo Choi, ${ }^{2}$ Ying Zhou, ${ }^{3,4}$ Mingkun Zhao, ${ }^{1,3}$ \\ Xianzhou Song, ${ }^{1}$ Xi Chen, ${ }^{4}$ Mirjana Maletić-Savatić, ${ }^{5-7}$ Timothy Palzkill, ${ }^{1,7}$ David Moore, ${ }^{2,4}$ \\ Meng C. Wang, ${ }^{8-10}$ and Jin Wang ${ }^{1,4,7}$
}

\begin{abstract}
Aims: Quantitative imaging of glutathione (GSH) with high spatial and temporal resolution is essential for studying the roles of GSH in redox biology. To study the long-standing question of compartmentalization of GSH, especially its distribution between the nucleus and cytosol, an organelle-targeted quantitative probe is needed.

Results: We developed a reversible reaction-based ratiometric fluorescent probe-HaloRT - that can quantitatively measure GSH dynamics with subcellular resolution in real time. Using HaloRT, we quantitatively measured the GSH concentrations in the nucleus and cytosol of HeLa cells and primary hepatocytes under different treatment conditions and found no appreciable concentration gradients between these two organelles. Innovation and Conclusion: We developed the first reversible ratiometric GSH probe that can be universally targeted to any organelle of interest. Taking advantage of this new tool, we provided definitive evidence showing that GSH concentrations are not significantly different between the nucleus and cytosol, challenging the view of nuclear compartmentalization of GSH. Antioxid. Redox Signal. 30, 1900-1910.
\end{abstract}

Keywords: glutathione, organelle-targeted probe, HaloTag, small-molecule fluorescent probe, compartmentalization of glutathione, live-cell imaging

\section{Introduction}

G LUTATHIONE (GSH), THE MOST abundant nonprotein small peptide in eukaryotic cells, regulates the intracellular redox homeostasis with its oxidized disulfide partner, oxidized glutathione. Imbalanced redox states have been shown to lead to various abnormal conditions such as diseases and drug resistance $(17,20)$. Understanding the intracellular concentration and distribution of GSH provides the most direct evidence to a localized cellular redox state and is the key step to study GSH-related redox biology. Just as pH is essential to all cellular events, GSH level is also crucial to a

\section{Innovation}

Currently, there is no glutathione (GSH) probe available that can target any organelle of interest. Our HaloRT probe is the first quantitative GSH probe that can be used to measure GSH levels in different organelles independent of the probe concentration. A long-standing debate in the field is whether the nucleus has higher GSH over the cytosol. Our observation showed that GSH concentrations are not significantly different in these two organelles, providing the first direct evidence challenging the view of nuclear compartmentalization of GSH.

Departments of ${ }^{1}$ Pharmacology and Chemical Biology, ${ }^{4}$ Molecular and Cellular Biology, ${ }^{5}$ Pediatrics, and ${ }^{8}$ Molecular and Human Genetics, Baylor College of Medicine, Houston, Texas.

${ }^{2}$ Program in Developmental Biology, Baylor College of Medicine, Houston, Texas.

${ }^{3}$ Integrative Molecular and Biomedical Sciences Graduate Program, Baylor College of Medicine, Houston, Texas.

${ }^{6} \mathrm{Jan}$ and Dan Duncan Neurological Research Institute at Texas Children's Hospital, Houston, Texas.

${ }^{7}$ Center for Drug Discovery, Baylor College of Medicine, Houston, Texas.

${ }^{9}$ Huffington Center on Aging, Baylor College of Medicine, Houston, Texas.

${ }^{10}$ Howard Hughes Medical Institute, Houston, Texas.

*These authors contributed equally to this work. 
considerable number of biochemical processes inside cells. Importantly, the activity of GSH is tightly linked to its spatial and temporal dynamics, which, however, remains poorly characterized due to lack of effective methods for quantifying subcellular GSH levels in real time.

Our group pioneered the use of reversible reaction-based ratiometric fluorescent probes for measuring GSH concentrations in live cells. We developed the first fluorescent probe ThiolQuant Green that can quantitatively measure intracellular GSH levels in live cells (10). Building on this work, we developed the second-generation GSH probe RealThiol (RT), which allows monitoring dynamic changes of GSH levels in real time (9). The groups of Urano and Yoon also independently reported two probes that can follow GSH dynamics $(11,18)$. Recently, we developed a mitochondria-targeted GSH probe, MitoRT (5), taking advantage of the high binding affinity of a triphenylphosphonium moiety toward the highly negatively charged mitochondrial membrane (16) and revealed the specificity of mitochondrial GSH dynamics. Unfortunately, this small-molecule-based subcellular targeting strategy is not suitable to all different organelles.

To develop a universal strategy that can be easily and broadly applied to any organelle of interest, we designed a HaloTag-enabled GSH probe, named HaloRT (Fig. 1a). In this study, using different signaling peptide sequences, we are able to specifically locate HaloRT in the nucleus, cytosol, mitochondria, and the endoplasmic reticulum (ER), and measure organellespecific GSH concentrations. To our surprise, we discovered that there is no appreciable GSH concentration gradient between the nucleus and cytosol in HeLa cells and in primary hepatocytes under several different treatment conditions, strongly challenging the existing view of a nuclear pool of GSH (7).

\section{Results and Discussion}

To achieve subcellular specificity for GSH probes, we adopted the widely used HaloTag technology for organellespecific protein tagging (12). Derived from our GSH RT probe, we substituted the dicarboxylic acid moiety with the HaloTag protein substrate elongated with a four-carbon linker (HaloRT ligand, Fig. 1a). Then, by expressing organellespecific HaloTag proteins in live cells, the HaloRT ligand can be recruited to different organelles and used for quantitative real-time imaging of GSH at subcellular resolution.

After successfully synthesizing the HaloRT ligand, we first verified that HaloRT, the conjugate between the HaloRT li- gand and recombinant HaloTag protein, exhibits similar GSH sensing abilities as RT. Similar to RT that has absorption maxima at 478 and $406 \mathrm{~nm}$ before and after reacting with GSH, respectively, HaloRT exhibits a bright orange color, an absorption maximum at $480 \mathrm{~nm}$, and shows fluorescence maximum at $565 \mathrm{~nm}$ on excitation at $488 \mathrm{~nm}$. Importantly, addition of GSH shifts the absorption and fluorescence peaks to 390 and $488 \mathrm{~nm}$ (excited at $405 \mathrm{~nm}$ ), respectively (Fig. 1b, c).

The kinetics between HaloRT and GSH is also very similar to that of RT, which typically equilibrates with physiologically relevant concentration of GSH within minutes. We measured the reaction using a stopped-flow rapid mixing device at room temperature and monitored the formation and disappearance of HaloRT-GSH by fluorescence $\left(\lambda_{\mathrm{ex}}=405 \mathrm{~nm}\right.$, $\left.\lambda_{\mathrm{em}}=480 \mathrm{~nm}\right)$. We observed that both forward and reverse reactions equilibrate in about $200 \mathrm{~s}$ (Fig. 1d). We also tested the reversibility of the sensing reaction by sequentially adding GSH and an irreversible thiol scavenger, $\mathrm{N}$ methylmaleimide (NMM), to the HaloRT ligand solution. As expected, the fluorescent signals from both blue and green channels as well as the ratio can reversibly reflect the corresponding GSH concentrations (Supplementary Fig. S1).

To measure the dissociation equilibrium constant $\left(K_{\mathrm{d}}\right)$ of the reversible sensing reaction, we mixed a series of known concentrations of GSH (from 0.39 to $50 \mathrm{mM}$ ) with HaloRT $(\sim 10 \mu M)$ and measured the fluorescence in two channels $\left(\lambda_{\text {ex } 1}=405 \mathrm{~nm}, \lambda_{\text {em } 1}=480 \mathrm{~nm} ; \lambda_{\text {ex } 2}=488 \mathrm{~nm}, \lambda_{\text {em } 2}=565 \mathrm{~nm}\right)$. The $K_{\mathrm{d}}$ for the sensing reaction is $121 \mathrm{~m} M$, 32-fold higher than that of RT.

The low reactivity of the protein-conjugated RT may be due to the steric hindrance of the protein or the hydrophobic HaloTag protein surface shifts the $K_{\mathrm{d}}$ of thiol-Michael addition reactions. It should be noted that the apparent dissociation equilibrium constant $\left(K_{\mathrm{d}}{ }^{\prime}\right)$, which is dependent on instrument settings, is more relevant in determining the dynamic range of the probe (please refer to the supporting information in reference 11 for detailed explanation). By adjusting the gains of both emission channels, $K_{\mathrm{d}}{ }^{\prime}$ values are 24.8 and $23.7 \mathrm{~m} M$ measured by a plate reader and a confocal microscope used in this study. In both cases, the calibration curve confers a superb linear relationship with $R^{2}$ above 0.99 within the physiologically relevant GSH concentration range (1.25-15 mM, Fig. 1e and Supplementary Fig. S2).

The high $K_{\mathrm{d}}$ of HaloRT results in a very small percentage of HaloRT-GSH formed under physiological conditions.

FIG. 1. Characterization of HaloRT probe. (a) Chemical structure of HaloRT ligand and the modular design diagram. (b) Reversible reaction between HaloRT and GSH. (c) Deconvoluted, normalized UV-vis absorption spectra (unshaded) and fluorescent spectra (shaded) of HaloRT (green, $\lambda_{\mathrm{ex}}=488 \mathrm{~nm}$ ) and HaloRT-GSH conjugate $\left(\right.$ blue, $\left.\lambda_{\mathrm{ex}}=405 \mathrm{~nm}\right)$. $(\mathbf{d}) \mathrm{Re}-$ action kinetics of HaloRT and GSH measured on a stopped-flow rapid mixing device with a fluorescence detector. Forward and backward reactions were monitored at $\lambda_{\mathrm{ex}}=405 \mathrm{~nm}, \lambda_{\mathrm{em}}=480 \mathrm{~nm}$, which correspond to the formation and dissociation of HaloRT-GSH conjugate. (e) Calibration curve determined by mixing a series of known concentrations of GSH with HaloRT, followed by imaging on a coverslip using confocal microscopy. The calculated $K_{\mathrm{d}}{ }^{\prime}$ is $23.7 \mathrm{~m} M$, and the $R^{2}$ is 0.991 in the physiologically relevant concentration range of GSH. (f) Selectivity of HaloRT probe in solution phase. Relative fluorescent ratio between blue and green channels was measured following mixing HaloRT probe with $\left.\mathrm{GSH}_{(10} \mathrm{m} M\right), \mathrm{H}_{2} \mathrm{O}_{2}$ $(100 \mu M), \mathrm{tBuOOH}(100 \mu M), \mathrm{Na}_{2} \mathrm{SO}_{3}(100 \mu M)$, lipoic acid $(100 \mu M)$, homocysteine $(100 \mu M)$, hydrogen sulfide $(100 \mu M)$, and cysteine $(100 \mu M)$. (g) Gel permeation chromatography trace of standard HaloRT-GSH conjugate and lysate from cytoHaloRT-stained HeLa cells. Fluorescent signal $\left(\lambda_{\mathrm{ex}}=405 \mathrm{~nm}, \lambda_{\mathrm{em}}=480 \mathrm{~nm}\right)$ was used for detecting HaloRT-GSH conjugate. Calculation of the area under the curve revealed that $87 \%$ of the signal can be attributed to HaloRT-GSH, indicating high selectivity toward GSH over protein thiols inside cells. FL, fluorescence; GSH, glutathione; $\mathrm{H}_{2} \mathrm{O}_{2}$, hydrogen peroxide; $\mathrm{RT}$, RealThiol probe. Color images are available online. 
a

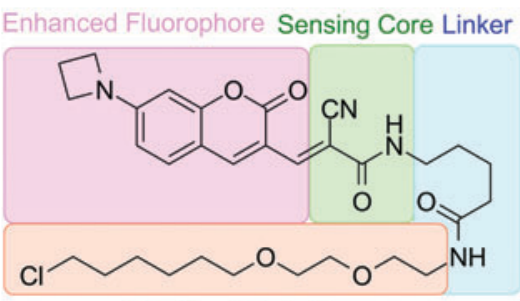

HaloTag Substrate

HaloRT Ligand

b

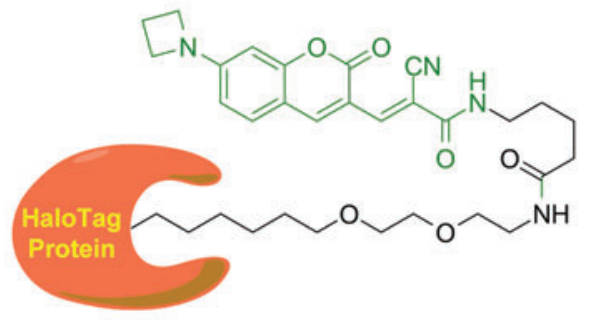

HaloRT Probe

(HaloRT Ligand + HaloTag Protein)

d
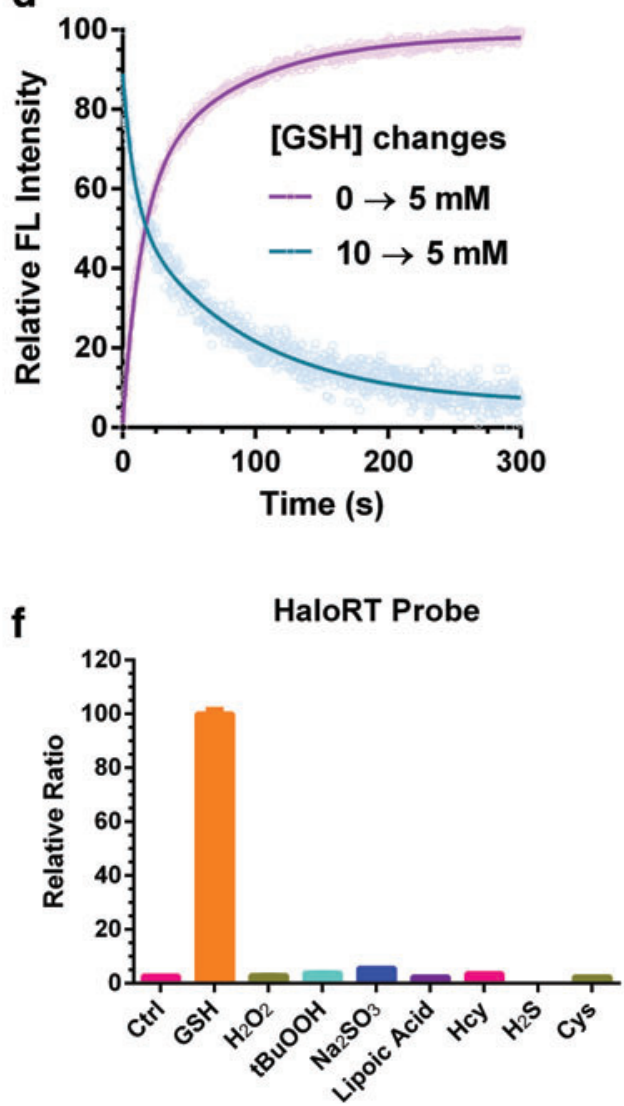

c

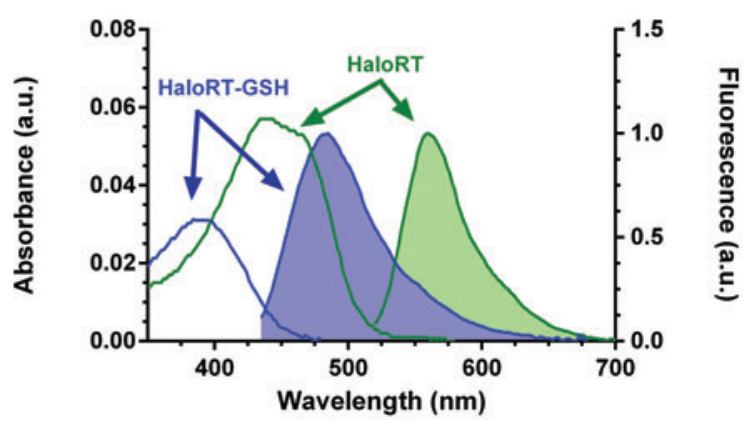

GSH

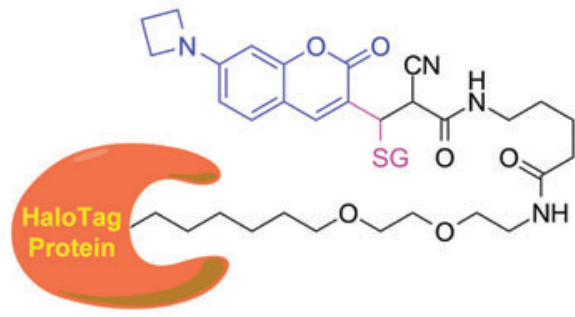

HaloRT-GSH

e

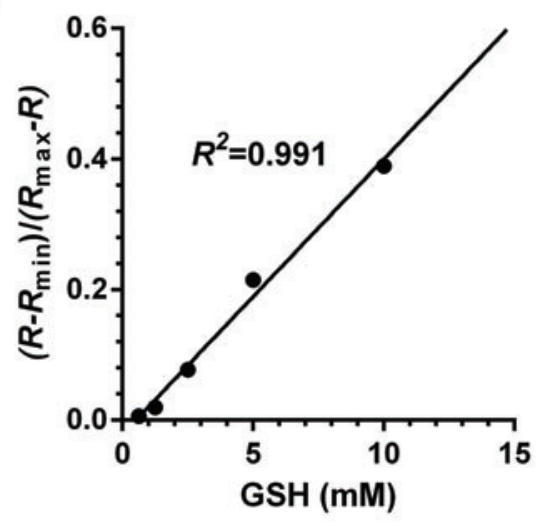

g

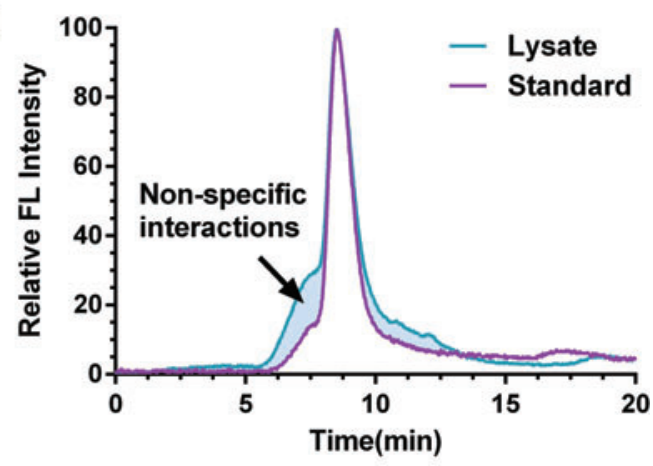


Compared with RT, we increased the gain for the $405 \mathrm{~nm}$ excitation channel to achieve sensitive detection of the subtle changes of HaloRT-GSH level. It should be noted that similar to RT, lower $\mathrm{pH}$ will alter the kinetics and thermodynamics of reaction between HaloRT and GSH. Thus, lysosomal GSH measurement using HaloRT may require recharacterization of the probe.

Next, we determined if the probe is selective toward GSH in cells. As shown in Figure 1f and Supplementary Figure S3, both HaloRT probe and HaloRT ligand only respond to physiologically relevant concentration of GSH but not to other biothiols or reactive oxygen species. Similar to the RT probe, we suggest validating measured GSH concentrations with other methods such as liquid chromatography-mass spectrometry to ensure that the GSH level is at least 10-fold higher than that of cysteine. In cases that GSH is lower than $1 \mathrm{~m} M$ and cysteine is still at its physiologically relevant concentration of $100 \mu \mathrm{M}$, the accuracy of GSH measurements based on HaloRT may be compromised.

We also applied the gel permeation chromatography (GPC) method with fluorescence detection that we developed for assessing the selectivity of RT in a complicated intracellular environment (9). The lysate of HaloRT-stained cells was washed using a denature buffer at $\mathrm{pH} 4.5$ to prevent reverse reactions, and the fluorescence signal of HaloRT-GSH $\left(\lambda_{\mathrm{ex}}=405 \mathrm{~nm}, \lambda_{\mathrm{em}}=480 \mathrm{~nm}\right)$ was used for detection. Compared with the HaloRT-GSH, standard underwent the same processing, we did not observe significant molecular-weight changes (Fig. 1g), suggesting that the HaloRT probe selectively reacts with small-molecule thiols but not protein thiols in cells. Together, based on the fluorescence spectra, kinetic and thermodynamic constants, and the selectivity profile, we conclude that HaloRT is suitable to quantitatively monitor GSH level changes under cellular conditions in real time.

To express the HaloTag protein in different organelles, we fused it with organelle targeting sequences, Halo-nuclear localization sequence (NLS) with a nuclear localization sequence, Halo-MapKK with a cytosolic localization sequence from MapK kinase (MapKK) (4), Halo-KDEL with an ER retention sequence, and Halo-MTS with a mitochondria targeting sequence. HeLa cells were transiently transfected with Halo-NLS, Halo-MapKK, Halo-KDEL, or Halo-MTS, incubated with the HaloRT ligand at $37^{\circ} \mathrm{C}$ for $30 \mathrm{~min}$ and then imaged using a confocal microscope. We confirmed that Halo-NLS, Halo-MapKK, Halo-KDEL, and Halo-MTS specifically target the HaloRT ligand to the nucleus (nucHaloRT), cytosol (cyto-HaloRT), endoplasmic reticulum (ER-HaloRT), and mitochondria (Mito-HaloRT), respectively (Fig. 2a). Thus, our strategy is effective to target the GSH probe to different cellular organelles.

It should be noted that our transient transfection protocol does not yield $100 \%$ transfection efficiency and the protein expression levels of the transfected cells may vary. The nontransfected cells can serve as an internal control showing that the HaloRT ligand will only stain transfected cells. As shown in Figure $2 \mathrm{~b}$, the cell at location 1 was completely nontransfected and the fluorescence signal was close to the background (Table 1). In contrast, the transfected cells at locations 2 and 3 showed significantly higher fluorescence signals than the background in both channels, indicating a successful transfection and staining. Due to the inhomogeneous protein expression levels, the absolute intensities from locations 2 and
3 varied by $\sim 3$-fold. While this may pose a great challenge for intensity-based quantification, it does not affect the final ratiometric readout of HaloRT (Table 1). The advantageous ratiometric quantification by HaloRT probe is clearly independent of the absolute probe concentration.

It is generally accepted that GSH is compartmentalized in different organelles and regulated separately there, but whether there is a nuclear pool of GSH remains a longstanding debate $(2,3,15)$. Bellomo et al. were the first to report a nuclear pool of GSH in primary hepatocytes using monochlorobimane (MCB) as a GSH probe (2). However, this study was challenged by Briviba et al. based on their observation that the MCB-GSH conjugate microinjected into the cytosol can preferentially accumulate in the nucleus, suggesting that MCB could react with cytosolic GSH (cGSH) first and the conjugate is further enriched in the nucleus (3).

Although some other studies favor the view of nuclear pool of GSH especially at early phases of cell proliferation, the fluorescent probes used suffer the same potential artifact as MCB (7, 13). It should be noted that 5-chloromethylfluorescein diacetate (CMFDA) was used as a GSH probe in several studies $(7,13)$. However, the fluorescence changes of CMFDA are due to fluorescein formation through esterase-mediated hydrolysis and independent of GSH conjugation. Therefore, it is questionable to use CMFDA as a GSH probe. Moreover, most previously studies using small-molecule GSH probes could not rule out the possibility that the irreversibly formed probe-GSH conjugate could diffuse through nuclear pore complex.

In our system, the readouts of HaloRT are independent of probe concentrations due to the ratiometric nature. The HaloNLS and Halo-MapKK are first transfected into the cells and expressed into their targeted organelles before seeing the HaloRT ligand. In addition, we have confirmed that nucHaloRT and cyto-HaloRT have high spatial specificity. Thus, we have applied our system to reexamine the nuclear GSH (nGSH) postulate.

We discovered no appreciable concentration gradient of GSH between the nucleus and cytosol. Despite the absolute fluorescence signals from the nucleus and cytosol being slightly different due to the variation of transfection efficiencies, the ratios of fluorescence signals remain statistically the same in these two locations. This ratiometric feature of our GSH probes is a unique advantage to avoid the nonspecific influence from different transfection efficiencies and probe availability (Fig. 3a, b).

Next, to investigate whether the nGSH can be better maintained compared with cGSH, we treated Halo-NLS and HaloMapKK cotransfected HeLa cells with different compounds and measured the corresponding nGSH and cGSH under these conditions. In a time-lapsed experiment, we treated cells with a bolus of $500 \mu M$ of hydrogen peroxide $\left(\mathrm{H}_{2} \mathrm{O}_{2}\right)$ to induce oxidative stress and imaged for 5 min consecutively. As shown in Figure 3c, both nGSH and cGSH levels decrease synchronously, but there is no significant GSH gradient between the nucleus and cytosol throughout this period. Further quantification demonstrates that $\mathrm{nGSH}$ and cGSH levels are not different from each other before and after $\mathrm{H}_{2} \mathrm{O}_{2}$ treatments (Fig. 3d).

We confirmed the quantification results with the same experiment performed using RT as a GSH probe (Supplementary Fig. S4). We also applied a non-GSH responsive HaloTag substrate, JF549, as our control and its fluorescence 


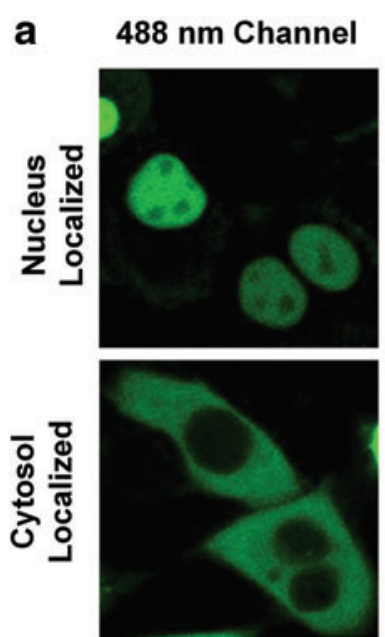

405 nm Channel
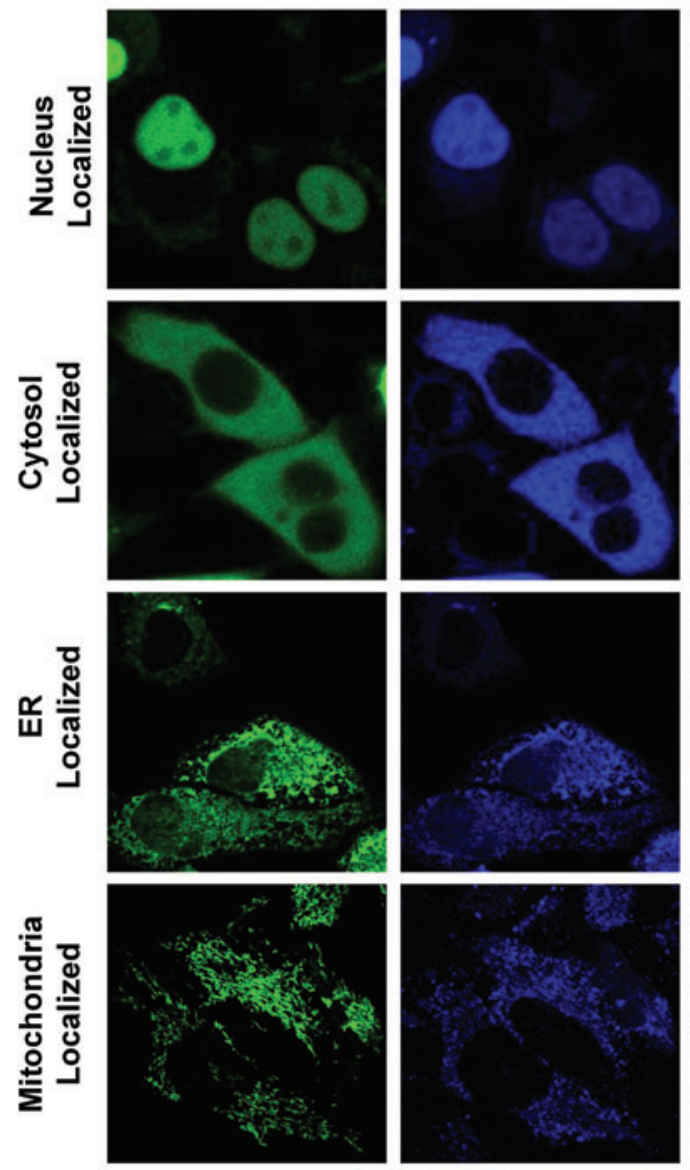

b
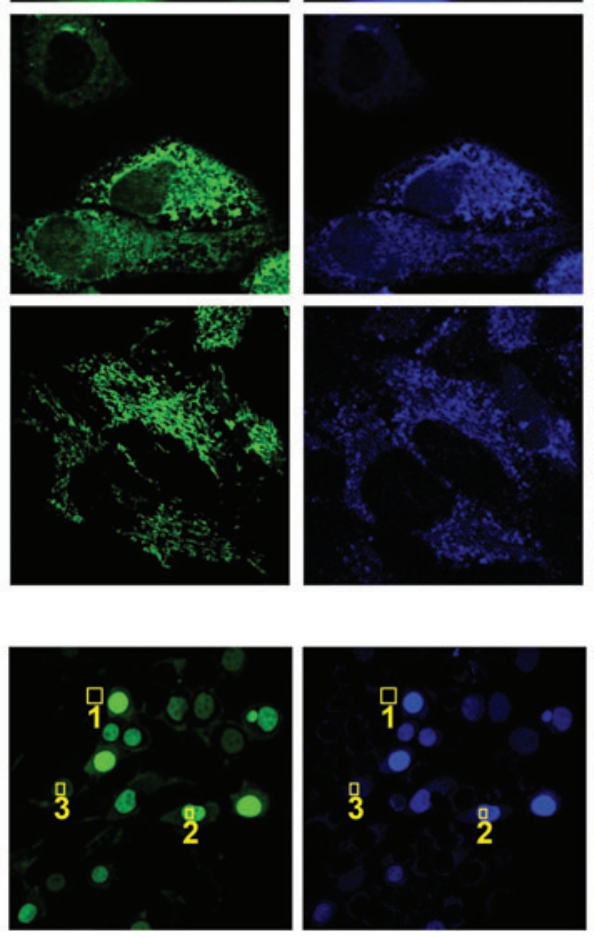
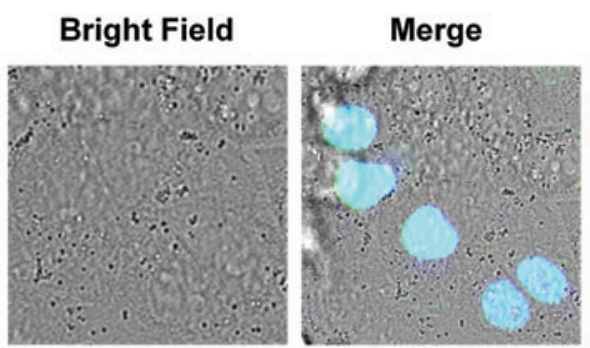

\section{Ratio Map}
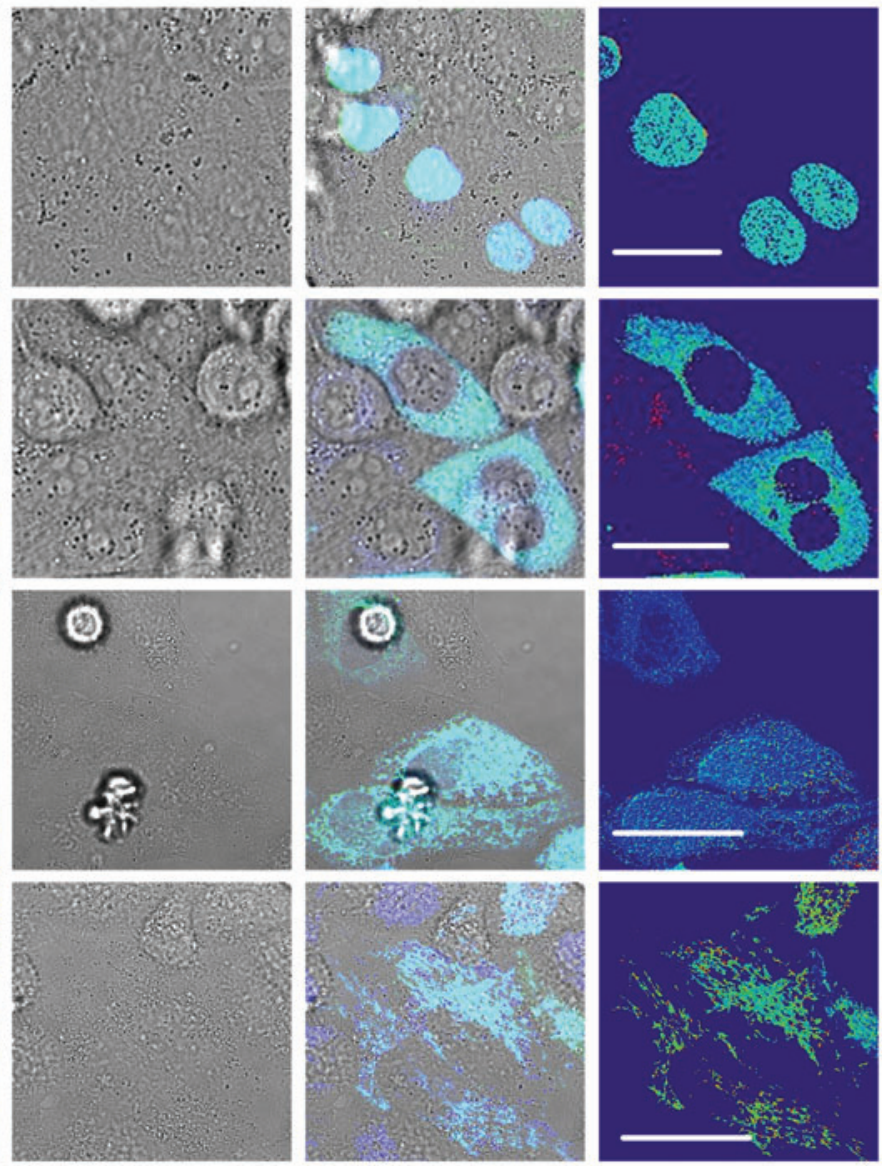

High

GSH

Low
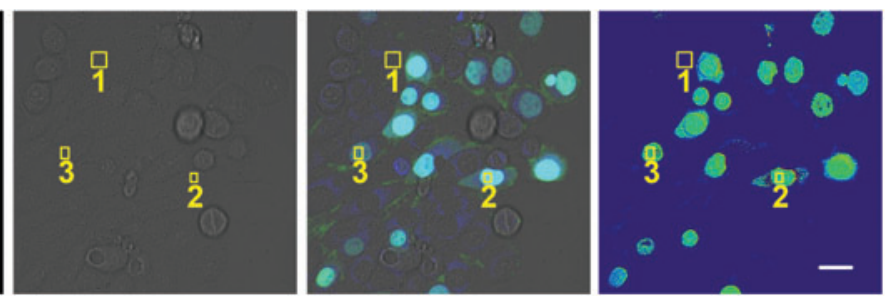

FIG. 2. Representative confocal images of HaloRT-stained HeLa cells. (a) Nuclear-, cytosolic-, ER-, and mitochondria-specific HaloTag expressing HeLa cells were stained with HaloRT and imaged with a confocal microscopy at blue and green channels sequentially. Ratio maps were calculated by dividing the blue channel by the green channel at each corresponding pixel. Scale bars are $10 \mu \mathrm{m}$. (b) Nuclear-specific HaloTag expressing HeLa cells were stained with HaloRT and quantitatively measured using a confocal microscopy. Position 1 illustrated a nontransfected cell, position 2 illustrated a high-expression transfected cell, and position 3 illustrated a low-expression transfected cell. The quantification results are presented in Table 1, showing the probe concentration independent ratiometric readouts. ER, endoplasmic reticulum. Color images are available online.

Table 1. Quantification Results of Figure 2B

\begin{tabular}{lccc}
\hline & \multicolumn{2}{c}{ Fluorescent intensity } & \\
\cline { 2 - 3 } Area & $\begin{array}{c}\text { Blue } \\
\text { channel }\end{array}$ & $\begin{array}{c}\text { Green } \\
\text { channel }\end{array}$ & $\begin{array}{c}\text { Ratio } \\
\text { (blue/green) }\end{array}$ \\
\hline Background & $125 \pm 60$ & $51 \pm 12$ & $\mathrm{NA}$ \\
1 & $118 \pm 95$ & $69 \pm 36$ & $\mathrm{NA}$ \\
2 & $3067 \pm 375$ & $3464 \pm 325$ & $0.893 \pm 0.109$ \\
3 & $949 \pm 210$ & $1070 \pm 136$ & $0.913 \pm 0.230$ \\
\hline
\end{tabular}

signal did not change on the same $\mathrm{H}_{2} \mathrm{O}_{2}$ treatment (Supplementary Fig. S5), indicating that the fluorescence signal changes of HaloRT are due to $\mathrm{H}_{2} \mathrm{O}_{2}$-induced GSH level changes.

We also challenged cells with other stressors and examined their effects on nGSH and cGSH. Buthionine sulfoximine (BSO) is a specific GSH synthase inhibitor that decreases intracellular GSH in a concentration-dependent manner. When we treated HeLa cells with 50 or $500 \mu M$ of BSO for 48 hours, we found that both nGSH and cGSH decrease as BSO concentration increases (Fig. 3e and Supplementary Fig. S6), but their levels are statistically the same. 


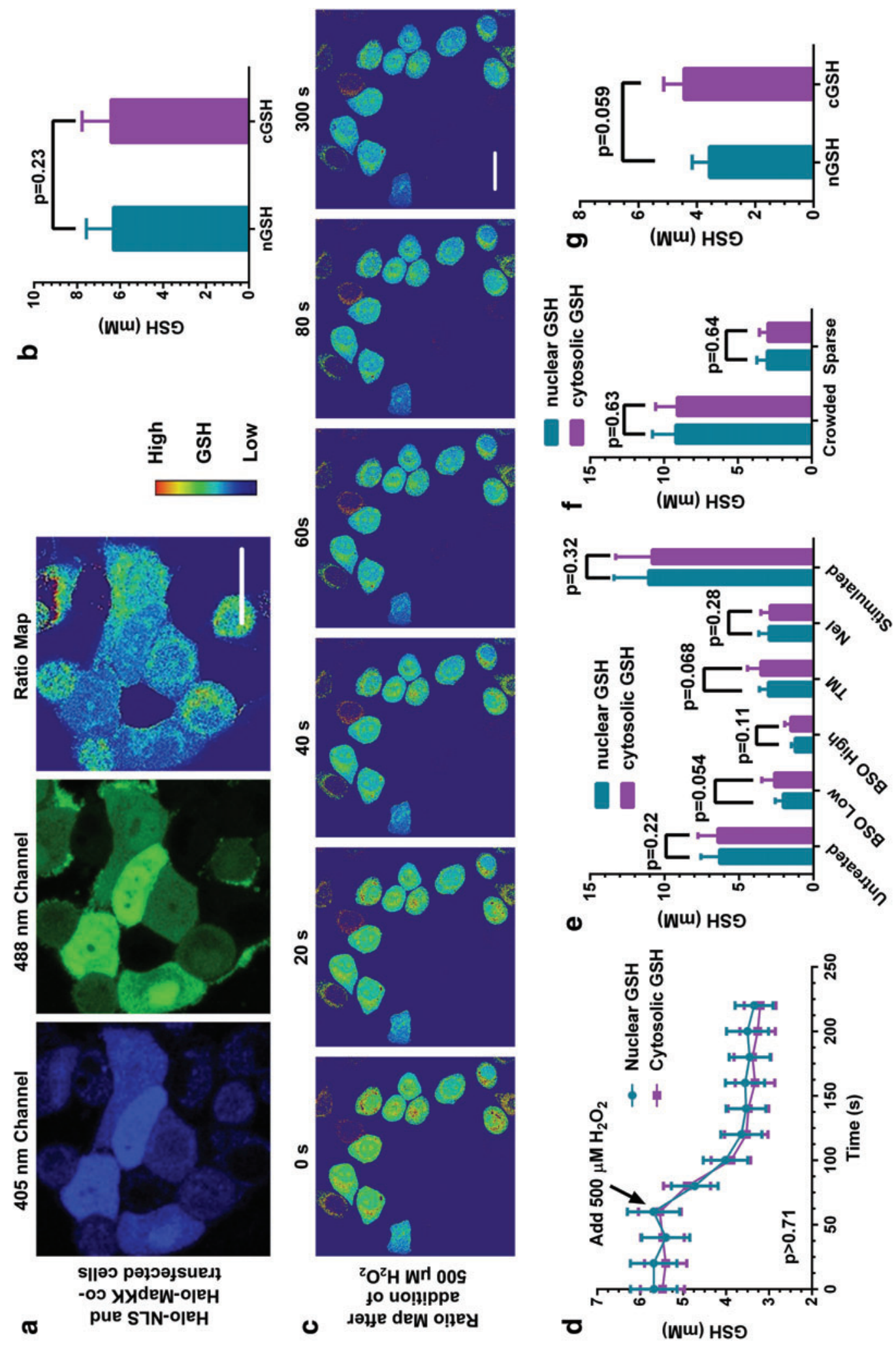

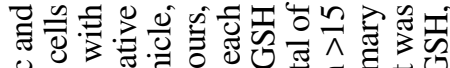

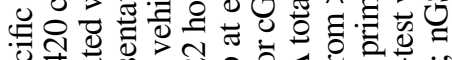

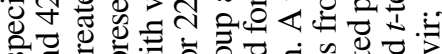

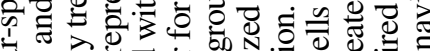

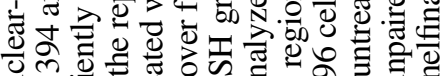

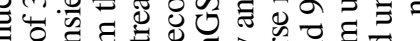

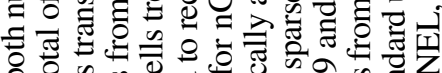

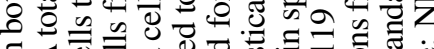

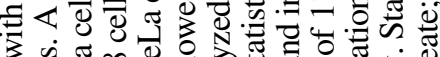

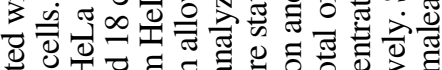

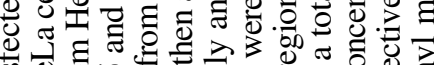

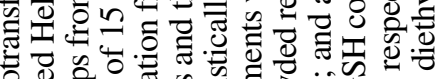

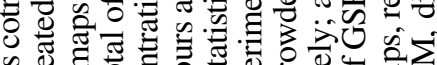

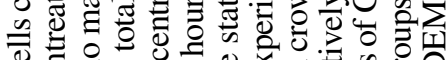

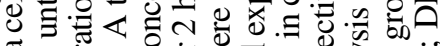

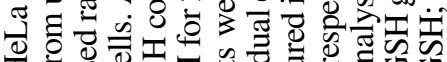

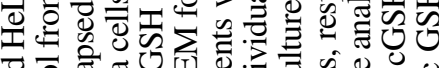

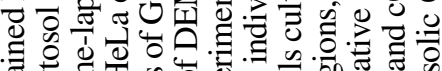

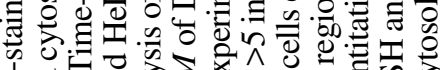

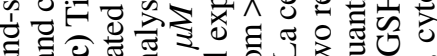

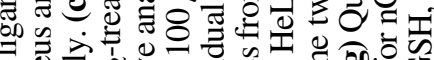

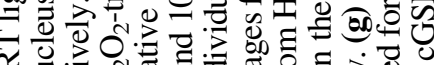

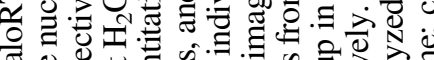

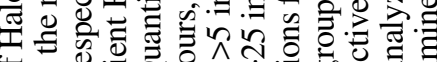

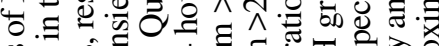

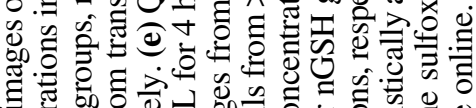

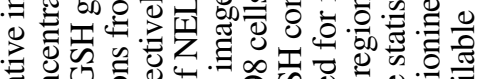

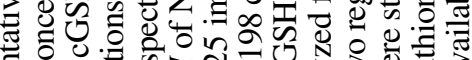

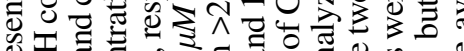

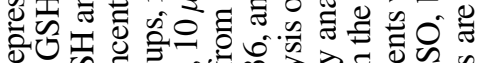
Q ङ.

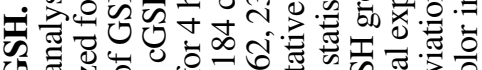

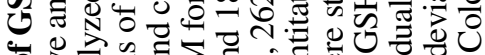

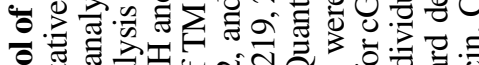

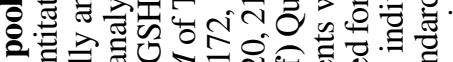

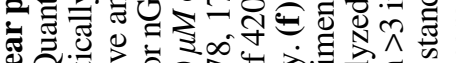

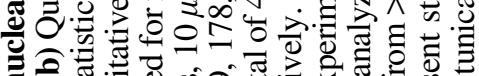

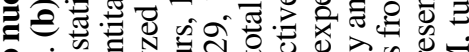

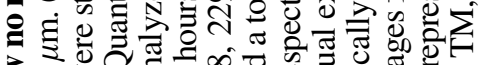

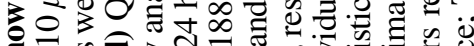

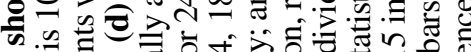

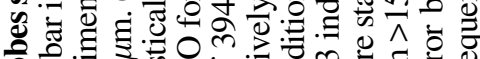

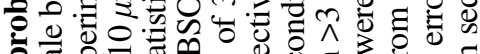
페

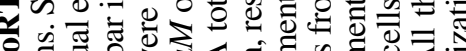

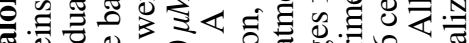

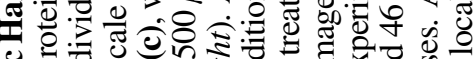

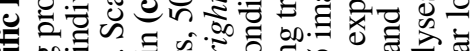

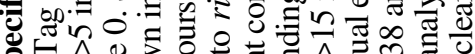

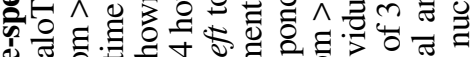

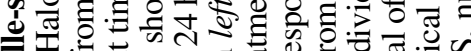
论 ton 00000

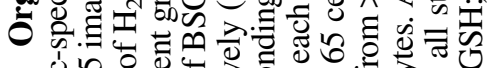

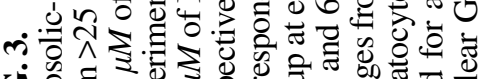

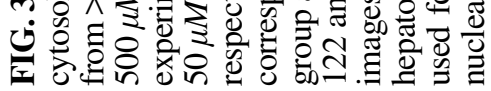


ER is a critical cellular compartment for protein folding, modification, and quality control, which is linked to the oxidizing balance of GSH. We thus have treated cells with tunicamycin (TM) and nelfinavir (NEL) to induce ER stress (14a) and test whether these treatments would affect nGSH and/or cGSH levels. We found that after 4 hours of treatments, nGSH and cGSH are decreased to similar levels (Fig. 3e), suggesting that ER stress-induced oxidative insults affect both the nucleus and cytosol. Together, these results show that shifting the oxidative balance toward oxidation reduces $\mathrm{nGSH}$ and cGSH levels at the same time and to the same degree, not supporting an independent pool of GSH in the nucleus.

On the contrary, we examined whether an increase in GSH would affect the concentration gradient between nGSH and cGSH. To this end, we stimulated cells with $100 \mu M$ of diethyl maleate (DEM) transiently for 2 hours and then allowed the cells to recover for 24 hours. We found that this treatment greatly increases GSH levels in the cell, but there is no difference between the nGSH and cGSH increases (Fig. 3e and Supplementary Fig. S6).

Densely cultured cells are known to have higher GSH levels compared with sparsely cultured counterparts $(8,14)$. We thus generated a cell culture condition where crowded and sparse regions coexist within the same culture dish and show two- to threefolds of GSH concentration difference (Supplementary Fig. S7). Under this physiological condition without exogenous stimulants, we did not observe an appreciable difference between nGSH and cGSH (Fig. 3f).

Furthermore, we also examined nGSH and cGSH levels in primary hepatocytes transfected with Halo-NLS and HaloMapKK and stained with the HaloRT ligand. Consistent with our observation in cell line studies, there is no appreciable difference between nGSH and cGSH in these primary cells despite a lower transfection efficiency, compared with HeLa cells, being observed (Fig. 3g and Supplementary Fig. S8). determine GSH levels in any organelle of interest with targeted HaloTag protein expression.

This HaloRT system enables us to quantify GSH concentrations with subcellular resolution in real time. Taking advantage of this new system, we revisited the nGSH postulate. Our measurements do not reveal any appreciable GSH concentration gradients between the cytosol and nucleus and thus do not support an independent pool of nGSH. Considering the nuclear pore complex has an inner diameter of $\sim 9-12 \mathrm{~nm}$ and typically allows free diffusion of molecules less than $60 \mathrm{kDa}(1$, 19), the small tripeptide GSH should freely diffuse through the nuclear pores. In organelles with enclosed membrane structures, GSH can be regulated separately, such as in the mitochondria, as demonstrated previously (5). In addition, our findings are consistent with a previous study using redox fluorescent proteins to measure GSH redox potentials in these organelles (5).

Although we did not observe any nuclear pool of GSH under our experimental conditions, we cannot rule out the possibility that the nuclear pores can be closed to restrict the free diffusion of GSH under other experimental conditions, thus creating a GSH concentration gradient. In addition, although demonstrated only for the nucleus, cytosol, ER, and mitochondria, with the HaloRT probe in hand, this system can be potentially applied to any organelle and will be a valuable addition to the redox biology toolbox.

\section{Materials and Methods}

\section{Chemical synthesis of HaloRT ligand}

All the chemicals were purchased from Sigma-Aldrich or Alfa Aesar unless otherwise specified. 3-Carbaldehyde-7azetidinylcoumarin was purchased from Ascendex Scientific, LLC. Tetrahydrofuran was distilled over sodium benzophenone ketyl, and $\mathrm{CH}_{2} \mathrm{Cl}_{2}$ was distilled over phosphorus pentoxide. All the other solvents and reagents were used as received without further purification.

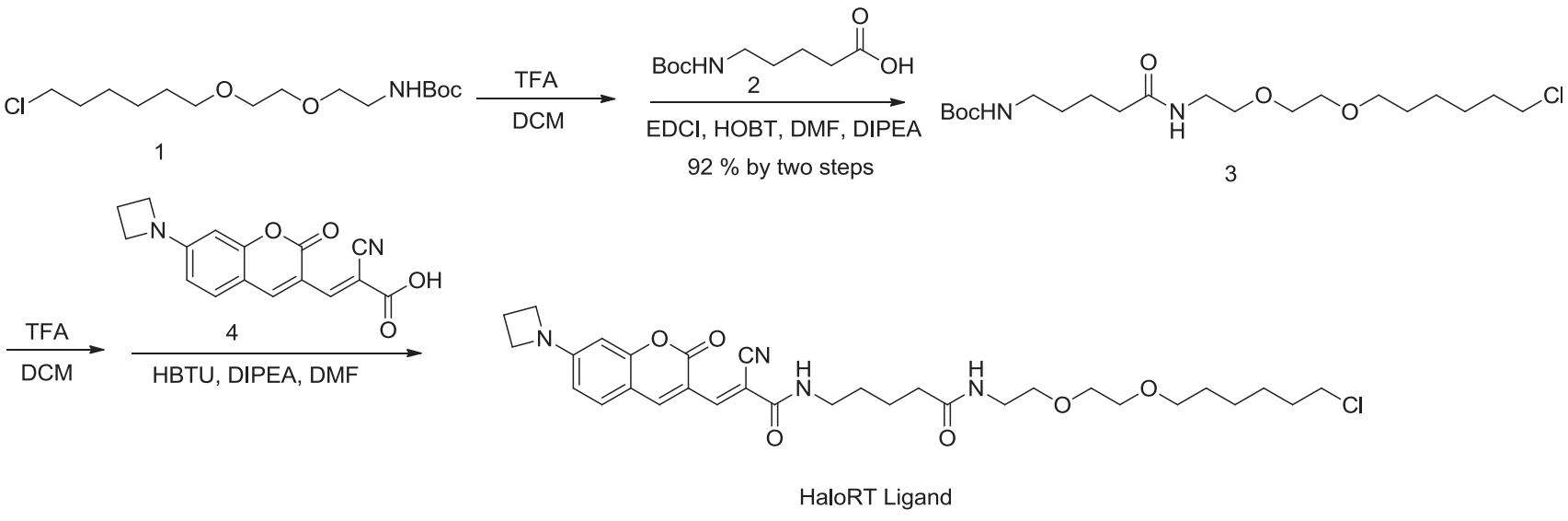

Therefore, we did not observe a significant concentration difference between nGSH and cGSH with increased GSH levels in HeLa cells and under physiological conditions in primary hepatocytes.

In conclusion, we have developed an organelle-specific GSH probe, HaloRT, which can be universally applied to
Tert-butyl (2-(2-((6-chlorohexyl)oxy)ethoxy)ethyl)carbamate (1, $65 \mathrm{mg}, 0.2 \mathrm{mmol}$ ) (refer to Supplementary Fig. S9 for $\left.{ }^{1} \mathrm{H}-\mathrm{NMR}\right)$ was dissolved in dichloromethane (DCM) $(2 \mathrm{~mL})$ and treated with trifluoroacetic acid (TFA) $(1 \mathrm{~mL})$ at room temperature for 2 hours. The reaction mixture was concentrated, dried, and used in the next step without further purification. 
The residue was dissolved in dimethylformamide (DMF) (2 mL). 5-((tert-butoxycarbonyl)amino)pentanoic acid (2, $43 \mathrm{mg}, 0.2 \mathrm{mmol})$, 1-ethyl-3-(3-dimethylaminopropyl)carbodiimide (38 mg, $0.3 \mathrm{mmol})$, hydroxybenzotriazole (41 mg, $0.3 \mathrm{mmol})$, and $\mathrm{N}, \mathrm{N}$-diisopropylethylamine (DIPEA) $(0.18 \mathrm{~mL}$, $1.0 \mathrm{mmol}$ ) were subsequently added at room temperature. After stirring overnight, the solvent was removed under vacuum. The residue was purified by silica gel column chromatography to give tert-butyl (5-((2-(2-((6-chlorohexyl)oxy)eth-oxy)ethyl)amino)5-oxopentyl)carbamate as a colorless oil $(\mathbf{3}, 78 \mathrm{mg}$, yield $92 \%$ by two steps).

${ }^{1} \mathrm{H} \mathrm{NMR}\left(400 \mathrm{MHz}, \mathrm{CDCl}_{3}\right): \delta 6.12(\mathrm{~s}, 1 \mathrm{H}), 4.72(\mathrm{~s}, 1 \mathrm{H})$, 3.62-3.52 (m, 8H), 3.47-3.43 (m, 4H), 3.12-3.09 (m, 2H), $2.20(t, J=7.4 \mathrm{~Hz}, 2 \mathrm{H}), 1.81-1.74(\mathrm{~m}, 2 \mathrm{H}), 1.71-1.58(\mathrm{~m}$, 4H), $1.55-1.34(\mathrm{~m}, 15 \mathrm{H})$ (Supplementary Fig. S10). ${ }^{13} \mathrm{C}$ NMR $\left(100 \mathrm{MHz}, \mathrm{CDCl}_{3}\right) \delta 172.72,156.05,79.05,71.25$, 70.25, 70.02, 69.83, 45.01, 39.98, 39.13, 35.98, 32.51, 29.53, 29.45, 28.43, 26.67, 25.41, 22.69 (Supplementary Fig. S11).

Tert-butyl (5-((2-(2-((6-chlorohexyl)oxy)ethoxy)ethyl) amino)-5-oxopentyl)carbamate $(3,21 \mathrm{mg}, 0.05 \mathrm{mmol})$ was dissolved in DCM $(2 \mathrm{~mL})$ and treated with TFA $(1 \mathrm{~mL})$ at room temperature for 2 hours. The reaction mixture was concentrated, dried, and used in the next step without further purification. The residue was dissolved in DMF (1 mL). 3-(7-(azetidin-1-yl)-2-oxo-2H-chro-men-3-yl)-2-cyano-acrylic acid (4, $15 \mathrm{mg}, 0.05 \mathrm{mmol}$ ), N,N,N',N'-tetramethyl-O-1H-benzotriazol-1-yl)uronium hexafluorophosphate $(38 \mathrm{mg}, 0.1 \mathrm{mmol})$, and DIPEA $(0.044 \mathrm{~mL}, 0.25 \mathrm{mmol})$ were added subsequently at room temperature. After stirring overnight, the solvent was removed under vacuum. The residue was purified by silica gel column chromatography to give 5-(3-(7-(azetidin-1yl)-2-oxo-2H-chromen-3-yl)-2-cyanoacrylamido)-N-(2-(2-((6chlorohexyl)oxy)ethoxy)ethyl)pentanamide as a red solid (HaloRT Ligand, $15 \mathrm{mg}$, yield $50 \%$ ).

${ }^{1} \mathrm{H}$ NMR $\left(400 \mathrm{MHz}, \mathrm{CDCl}_{3}\right) \delta 8.80(\mathrm{~s}, 0.5 \mathrm{H}), 8.64(\mathrm{~s}$, $0.5 \mathrm{H}), 8.47(\mathrm{~s}, 0.5 \mathrm{H}), 7.65(\mathrm{~s}, 0.5 \mathrm{H}), 7.34(t, J=7.9 \mathrm{~Hz}, 1 \mathrm{H})$, $6.78(t, J=4.4 \mathrm{~Hz}, 0.5 \mathrm{H}), 6.53(t, J=4.8 \mathrm{~Hz}, 0.5 \mathrm{H}), 6.31-$ $6.15(\mathrm{~m}, 2 \mathrm{H}), 6.08(d, J=9.7 \mathrm{~Hz}, 1 \mathrm{H}), 4.14-4.03(\mathrm{~m}, 4 \mathrm{H})$, $3.62-3.47(\mathrm{~m}, 8 \mathrm{H}), 3.46-3.35(\mathrm{~m}, 6 \mathrm{H}), 2.52-2.43(\mathrm{~m}, 2 \mathrm{H})$, $2.22(q, J=7.0 \mathrm{~Hz}, 2 \mathrm{H}), 1.78-1.58(\mathrm{~m}, 8 \mathrm{H}), 1.49-1.34(\mathrm{~m}, 4 \mathrm{H})$ (Supplementary Fig. S12). ${ }^{13} \mathrm{C}$ NMR $\left(100 \mathrm{MHz}, \mathrm{CDCl}_{3}\right) \delta$ $172.86,172.78,161.42,161.11,160.63,157.40,155.01$, $154.90,148.28,145.69,143.92,131.71,131.52,118.80$, $117.61,111.95,111.78,109.19,109.04,108.71,108.58$, 106.00, 101.68, 95.65, 95.55, 77.24, 71.27, 70.24, 70.21, 70.01, 69.99, 69.81, 69.79, 51.33, 45.05, 39.92, 39.73, 39.22, $35.75,32.52,32.51,29.43,28.86,28.63,26.68,25.40,25.39$, 22.63, 22.57, 16.15 (mixture of cis and trans isomers) (Supplementary Fig. S13). ESI $m / z=601.3[\mathrm{M}+\mathrm{H}]^{+}$.

\section{Synthesis of HaloRT probe}

HaloRT ligand $(400 \mu M, 200 \mu \mathrm{L})$ was incubated with purified His-HaloTag protein (Promega, G4491, $6.5 \mathrm{mg} / \mathrm{mL}$, $50 \mu \mathrm{L}$ ) for 1 hour at room temperature in phosphate-buffered saline (PBS). The reaction mixture was then concentrated with a 10k cutoff viva-spin 500 centrifugation tube and washed twice with fresh PBS. The resulting bright orange supernatant was then used as HaloRT probe for further testing. The HaloRT probe was validated by GPC to ensure that no free HaloRT ligand remained in solution.

\section{UV-Vis and fluorescence spectra of HaloRT probe}

HaloRT probe was diluted with PBS to $10 \mu M$ and mixed with an equal volume of PBS or GSH $(700 \mathrm{mM})$. The resulting mixture was then measured on a BioTek H1 plate reader for absorption and fluorescence at corresponding wavelengths using a Take3 Trio microvolume plate. A deconvolution of the overlapping spectra was performed to yield the individual spectrum.

\section{Kinetics of HaloRT probe reacting with GSH}

The reaction kinetics was measured on a stopped-flow rapid mixing device with a fluorescence detector. The formation and dissociation of HaloRT-GSH conjugate were monitored at $\lambda_{\mathrm{ex}}=405 \mathrm{~nm}$ and $\lambda_{\mathrm{em}}=480 \mathrm{~nm}$. The forward reaction was measured by mixing an equal volume of $10 \mu M$ HaloRT probe with $10 \mathrm{~m} M$ of GSH and the reverse reaction was measured by diluting a pre-equilibrated $10 \mu M$ HaloRT probe and $10 \mathrm{~m} M$ GSH mixture with an equal volume of PBS.

\section{Reversibility of HaloRT ligand reacting with GSH}

HaloRT ligand was diluted with PBS to $10 \mu M$ and monitored using a fluorimeter at $\lambda_{\mathrm{ex}}=405 \mathrm{~nm}, \lambda_{\mathrm{em}}=480 \mathrm{~nm}$ and $\lambda_{\text {ex }}=488 \mathrm{~nm}, \lambda_{\text {em }}=565 \mathrm{~nm}$. To $1 \mathrm{~mL}$ of the HaloRT ligand solution was added $50 \mu \mathrm{L}$ of $100 \mathrm{~m} M \mathrm{GSH}$ solution. After the reaction equilibrated ( $\sim 300 \mathrm{~s}$ ), $50 \mu \mathrm{L}$ of $100 \mathrm{~m} M$ NMM solution was added to the mixture. GSH concentration reflected by the ratio was confirmed with standard GSH solution with $>99 \%$ accuracy.

\section{Equilibrium constant of the reaction between HaloRT probe and GSH}

HaloRT probe was diluted with PBS to $10 \mu M$ and mixed with an equal volume of a series of known concentrations of $\operatorname{GSH}(0.39,0.78,1.56,3.13,6.25,12.5,25,50,100 \mathrm{mM})$ and equilibrated for $5 \mathrm{~min}$ at room temperature. The resulting mixture was then measured on a plate reader at two fluorescent channels: $\lambda_{\mathrm{ex}}=405 \mathrm{~nm}, \lambda_{\mathrm{em}}=480 \mathrm{~nm}$ and $\lambda_{\mathrm{ex}}=488 \mathrm{~nm}$, $\lambda_{\mathrm{em}}=565 \mathrm{~nm}$. The ratio between the two channels was used for calibration of equilibrium constant.

Similarly, the mixture of HaloRT probe and GSH was prepared and imaged using a confocal microscope. Before imaging, the mixture was mixed with $15 \mu \mathrm{m}$ polystyrene beads for focusing purpose. A typical sample can be prepared by sealing $5 \mu \mathrm{L}$ of solution with a coverslip.

\section{Selectivity of HaloRT ligand and HaloRT probe in solution}

HaloRT ligand and HaloRT probe were diluted with PBS to $10 \mu M$ and mixed with equal volumes of PBS, GSH $(20 \mathrm{~m} M), \quad \mathrm{H}_{2} \mathrm{O}_{2}(200 \mu M)$, tBuOOH $(200 \mu M), \quad \mathrm{Na}_{2} \mathrm{SO}_{3}$ $(200 \mu M)$, lipoic acid $(200 \mu M)$, homocysteine $(200 \mu M)$, hydrogen sulfide $(200 \mu M)$, and cysteine $(200 \mu M)$, respectively. The resulting mixture was then measured on plate reader at two fluorescent channels: $\lambda_{\mathrm{ex}}=405 \mathrm{~nm}, \lambda_{\mathrm{em}}=480$ $\mathrm{nm}$ and $\lambda_{\mathrm{ex}}=488 \mathrm{~nm}, \lambda_{\mathrm{em}}=565 \mathrm{~nm}$. The ratio between the two channels was used for calculation.

\section{Intracellular selectivity of HaloRT probe measured with GPC-fluorescence}

To acquire enough amount of material for analysis, we choose to overexpress HaloTag protein in the cytosol, which 
has the highest overall quantity of protein. HeLa cells (from a confluent $15 \mathrm{~mm}$ dish) transiently transfected with cytosolicspecific HaloTag protein were stained with $5 \mu M$ of HaloRT ligand at $37^{\circ} \mathrm{C}$ for $30 \mathrm{~min}$. The cells were washed twice with cold PBS to remove excessive small-molecule probes and then lysed using $1 \mathrm{~mL}$ of $10 \%$ trichloroacetic acid on ice. The lysate was then centrifuged at $15,000 \mathrm{~g}$ at $4^{\circ} \mathrm{C}$ for $15 \mathrm{~min}$.

The supernatant was ready for GPC-fluorescence (FL) measurement as the small-molecule mixture. The resulting precipitate was then redissolved using $0.1 \mathrm{MpH} 4.5$ citric acid-buffered $5 \% \mathrm{SDS}$ at $58^{\circ} \mathrm{C}$ for $5 \mathrm{~min}$. Supernatant was then collected after quickly centrifuging at 15,000 $\mathrm{g}$ at room temperature for $1 \mathrm{~min}$. This second supernatant was ready for GPC-FL measurement as the redissolved protein mixture.

The GPC-FL measurement was performed using $0.1 M \mathrm{pH}$ 4.5 citric acid-buffered $0.1 \%$ SDS solution with a cationic column. Flow rate was set at $1.5 \mathrm{~mL} / \mathrm{min}$ and the temperature was set at $45^{\circ} \mathrm{C}$. A standard HaloRT-GSH sample, a smallmolecule mixture from cell lysate, and a redissolved protein mixture from the same cell lysate were measured on GPC-FL with a fluorescent detector set at $\lambda_{\mathrm{ex}}=405 \mathrm{~nm}, \lambda_{\mathrm{em}}=480 \mathrm{~nm}$ for detection of HaloRT-GSH conjugate. The small-molecule mixture resulted in no fluorescent signal, and the HaloRT-GSH standard and the redissolved protein mixture showed similar retention time on their fluorescent signal. Area under the curve after normalization was used for quantification purpose. Analysis revealed that $87 \%$ of the protein mixture signal comes from HaloRT-GSH compared with the standard.

\section{General cell culture}

The HeLa cell line used in this study was purchased from American Type Culture Collection (ATCC) and grown in DMEM (11965; Gibco) supplemented with 10\% FBS and 1\% 1003 penicillin/streptomycin (Gibco). Cells were cultured under a controlled atmosphere $\left(37^{\circ} \mathrm{C}, 5 \% \mathrm{CO}_{2}\right)$. Glassbottomed dishes were used for cell culture due to confocal scanning requirements.

\section{Organelle-specific quantitative imaging of GSH using HaloRT probe}

Halo-NLS, Halo-MapKK, Halo-KDEL, and Halo-MTS plasmids were constructed by integrating organelle-specific signal sequences into the Halo cDNA sequence. The following signal sequences are used for plasmid constructs: $5^{\prime}$ CCAAAAAAGAAGAGAAAGGTAGAAGACCCC-3' for nuclear specific, 5'-TTGCAGAAGAAGCTGGAGGAGCT AGAGCTTGAT-3' for cytoplasm specific, 5'-AAAGATGA GCTATAA-3' (KDEL sequence) for ER specific, and $5^{\prime}$ ATGGCCTCCACTCGTGTCCTCGCCTCTCGCCTGGCC TCCCAGATGGCTGCTTCCGCCAAGGTTGCCCGCCCT GCTGTCCGCGTTGCTCAGGTCAGCAAGCGCACCAT CCAGACTGGCTCCCCCCTCCAGACCCTCAAGCGCA CCCAGATGGCCACCATGAGCGCCCTGCTGATCCTG GCTCTCGTGGGCGCTGCCGTGGCC-3' for mitochondria specific.

Whole piece of DNA fragment, including signal sequence and Halo cDNA sequence, was synthesized by GenScript and then inserted into pCDNA $3.1(+)$ vector through KpnI (5'end) and NotI ( $3^{\prime}$ end) sites.

HeLa cells $\left(4 \times 10^{5}\right)$ were seeded on a $35-\mathrm{mm}$ glassbottomed culture dish and incubated for 24 hours. The cells were then transfected with one or two plasmids using Lipofectamine 3000 transfection agent and incubated for an additional 36 hours. Before imaging, cells were stained with $1 \mu M$ HaloRT ligand in culture medium at $37^{\circ} \mathrm{C}$ for $30 \mathrm{~min}$ and then washed with fresh medium twice, each for $10 \mathrm{~min}$. Imaging was performed using a Zeiss LSM 780 confocal microscope with a temperature control chamber. Green channel signal was acquired with $\lambda_{\mathrm{ex}}=488 \mathrm{~nm}, \lambda_{\mathrm{em}}=499-$ $615 \mathrm{~nm}$; blue channel signal was acquired with $\lambda_{\mathrm{ex}}=405 \mathrm{~nm}$, $\lambda_{\mathrm{em}}=418-495 \mathrm{~nm}$.

The usage of transient transfection for all our experiments inevitably leads to nonhomogeneous transfection efficiency. Some cells are not transfected, while other cells are overexpressing the transfected plasmids. This inhomogeneous transfection resulted in inhomogeneous fluorescent signal from cell to cell. Theoretically, ratiometric imaging will not be affected by the differences in absolute fluorescent signal, but the instrument dynamic range is only accurate within limited range. For quantitation purpose, we have to compromise and use the settings that we are able to take the calibration curve with. Thus, for quantitative analysis, we only picked cells with $\mathrm{S} / N>5$ signals from both channels and do not have saturating pixels. In addition, because we could hardly define the boundary of nucleus from the ratio map in Halo-NLS and Halo-MapKK cotransfected cells, we manually labeled the nucleus and cytosol by comparing with the images from other channels.

\section{Treatment conditions for HeLa cells}

Transient treatment with $\mathrm{H}_{2} \mathrm{O}_{2}$ was performed at the site of imaging. Stained, transfected HeLa cells were treated with a bolus of $500 \mu M$ of $\mathrm{H}_{2} \mathrm{O}_{2}\left(100 \mu \mathrm{L} 5 \mathrm{mM} \mathrm{H} \mathrm{H}_{2} \mathrm{O}_{2}\right.$ in PBS diluted to $1 \mathrm{~mL}$ medium) in the imaging chamber and imaged at $37^{\circ} \mathrm{C}$ continuously for $5 \mathrm{~min}$ with $20 \mathrm{~s}$ per scan. This condition was designed for testing rapid GSH consumption after a bolus of external oxidative insult. The same $\mathrm{H}_{2} \mathrm{O}_{2}$ treatment was also applied to transfected cells stained with both HaloRT and JF549.

BSO (50 and $500 \mu M)$ was added to HeLa cells at seeding, and the cells were incubated for 24 hours before transfection of cells. Cells were imaged 48 hours after transfection. BSO is a GSH synthase inhibitor. This condition was designed for decreasing overall GSH concentrations in a controlled, dosedependent manner.

DEM $(100 \mu M)$ was added to HeLa cells 10 hours after transfection. Cells were incubated with DEM for 2 hours and then washed with fresh culture medium. Then, the cells were incubated for an additional 22 hours in fresh culture medium before imaging. This condition was designed for increasing GSH concentration.

ER stressors (10 $\mu M$ of TM and $10 \mu M$ of NEL) were added to cells 4 hours before imaging. This condition was designed to induce ER stress, which will transiently decrease GSH concentration.

HeLa cells were seeded on a confined spot on a culture dish without stirring, which created a crowded region. The peripheral of the crowded cells was sparse regions. This condition was designed for creating high- and lowconcentration GSH in cells on the same plate but at different regions. 


\section{Primary hepatocyte imaging}

Mice were euthanized with isoflurane and $25 \mathrm{G}$ needle was inserted into the inferior vena cava. After their portal vein is cut, peristaltic pump was used to perfuse the liver. First, Earle's balanced salt solution (EBSS) (14155063; Invitrogen) containing $5 \mathrm{~m} M$ EGTA was used. Following EBSS perfusion, Hank's balanced salt solution (24020117; Invitrogen) containing $100 \mathrm{U} / \mathrm{mL}$ collagenase and trypsin inhibitor was used. The liver was then harvested and massaged to obtain dissociated cells in hepatocyte wash medium (17704024; Invitrogen).

After cells went through mesh, the cells were sublayered onto Percoll (P4937; Sigma). Only viable cells at the bottom layer were washed twice with wash medium, and then, the cells were plated onto dishes in Wiliams E medium (12551; Invitrogen) containing insulin/transferrin/selenium supplementation. Then, the cells were kept in DMEM overnight. Transfection, staining, and confocal imaging were performed following the same procedure for HeLa cells.

\section{Data availability}

All relevant data are available from the authors.

\section{Acknowledgments}

The research was supported, in part, by the National Institutes of Health (R01-GM115622, R01-CA207701, R21CA213535, and RF1-AG062257 to J.W., R01-AG045183, R01-AT009050, R21-EB022302, DP1-DK113644, and RF1AG062257 to M.C.W., R01-GM120033 to M.M-S.), the Welch Foundation (Q-1912 to M.C.W.), Cancer Prevention and Research Institute of Texas (RP130573 to M.M.S.), the IDDRC Microscopy Core (P30HD024064 and 1U54 HD083092 Intellectual and Developmental Disabilities Research Grant from the Eunice Kennedy Shriver National Institute of Child Health and Human Development), the Optical Imaging and Vital Microscopy Core, and the Cytometry and Cell Sorting Core at Baylor College of Medicine with funding from the NIH (AI036211, CA125123, and RR024574), and the expert assistance of Joel M. Sederstrom. We also thank Dr. Lavis for providing the JF549 HaloTag substrate and the Department of Pharmacology and Chemical Biology at Baylor College of Medicine for providing partial support for the NMR.

\section{Authors' Contributions}

X.J., J.C., X.C., M.M-S., T.P., D.M., M.C.W., and J.W. designed the experiments; X.J., J.C., C.Z., S.C., Y.Z., M.Z., and X.S. performed the experiments; X.J., M.C.W., and J.W. performed data analysis and wrote the article.

\section{Author Disclosure Statement}

X.J., J.C., and J.W. are coinventors of a patent application related to this work.

\section{References}

1. Beck M, Lučić V, Förster F, Baumeister W, and Medalia O. Snapshots of nuclear pore complexes in action captured by cryo-electron tomography. Nature 449: 611-615, 2007.
2. Bellomo G, Vairetti M, Stivala L, Mirabelli F, Richelmi P, and Orrenius S. Demonstration of nuclear compartmentalization of glutathione in hepatocytes. Proc Natl Acad Sci U S A 89: 4412-4416, 1992.

3. Briviba K, Fraser G, Sies H, and Ketterer B. Distribution of the monochlorobimane-glutathione conjugate between nucleus and cytosol in isolated hepatocytes. Biochem J 294: 631-633, 1993.

4. Cambronne XA, Stewart ML, Kim D, Jones-Brunette AM, Morgan RK, Farrens DL, Cohen MS, and Goodman RH. Biosensor reveals multiple sources for mitochondrial $\mathrm{NAD}^{+}$. Science 352: 1474-1477, 2016.

5. Chen J, Jiang X, Zhang C, MacKenzie KR, Stossi F, Palzkill $\mathrm{T}$, Wang MC, and Wang J. Reversible reaction-based fluorescent probe for real-time imaging of glutathione dynamics in mitochondria. ACS Sens 2: 1257-1261, 2017.

6. This reference has been deleted.

7. García-Giménez JL, Markovic J, Dasí F, Queval G, Schnaubelt D, Foyer CH, and Pallardó FV. Nuclear glutathione. Biochim Biophys Acta 1830: 3304-3316, 2013.

8. Gutscher M, Pauleau A-L, Marty L, Brach T, Wabnitz GH, Samstag Y, Meyer AJ, and Dick TP. Real-time imaging of the intracellular glutathione redox potential. Nat Methods 5: 553-559, 2008.

9. Jiang X, Chen J, Bajić A, Zhang C, Song X, Carroll SL, Cai Z-L, Tang M, Xue M, Cheng N, Schaaf CP, Li F, MacKenzie KR, Ferreon ACM, Xia F, Wang MC, MaletićSavatić M, and Wang J. Quantitative real-time imaging of glutathione. Nat Commun 8: 16087, 2017.

10. Jiang X, Yu Y, Chen J, Zhao M, Chen H, Song X, Matzuk AJ, Carroll SL, Tan X, Sizovs A, Cheng N, Wang MC, and Wang J. Quantitative imaging of glutathione in live cells using a reversible reaction-based ratiometric fluorescent probe. ACS Chem Biol 10: 864-874, 2015.

11. Liu Z, Zhou X, Miao Y, Hu Y, Kwon N, Wu X, and Yoon J. A reversible fluorescent probe for real-time quantitative monitoring of cellular glutathione. Angew Chem Int Ed Engl 56: 5812-5816, 2017.

12. Los GV, Encell LP, McDougall MG, Hartzell DD, Karassina N, Zimprich C, Wood MG, Learish R, Ohana RF, Urh M, Simpson D, Mendez J, Zimmerman K, Otto P, Vidugiris G, Zhu J, Darzins A, Klaubert DH, Bulleit RF, and Wood KV. HaloTag: a novel protein labeling technology for cell imaging and protein analysis. ACS Chem Biol 3: 373-382, 2008.

13. Markovic J, Borrás C, Ortega A, Sastre J, Viña J, and Pallardó FV. Glutathione is recruited into the nucleus in early phases of cell proliferation. J Biol Chem 282: 2041620424, 2007.

14. Pani G, Colavitti R, Bedogni B, Anzevino R, Borrello S, and Galeotti T. A redox signaling mechanism for densitydependent inhibition of cell growth. J Biol Chem 275: 38891-38899, 2000.

14a. Peñaranda Fajardo NM, Meijer C, and Kruyt FAE. The endoplasmic reticulum stress/unfolded protein response in gliomagenesis, tumor progression and as a therapeutic target in glioblastoma. Biochem Pharmacol 118: 1-8, 2016.

15. Smith CV, Jones DP, Guenthner TM, Lash LH, and Lauterburg BH. Compartmentation of glutathione: implications for the study of toxicity and disease. Toxicol Appl Pharmacol 140: 1-12, 1996.

16. Smith RA, Porteous CM, Gane AM, and Murphy MP. Delivery of bioactive molecules to mitochondria in vivo. Proc Natl Acad Sci U S A 100: 5407-5412, 2003. 
17. Townsend DM, Tew KD, and Tapiero H. The importance of glutathione in human disease. Biomed Pharmacother 57: 145-155, 2003.

18. Umezawa K, Yoshida M, Kamiya M, Yamasoba T, and Urano Y. Rational design of reversible fluorescent probes for live-cell imaging and quantification of fast glutathione dynamics. Nat Chem 9: 279-286, 2017.

19. Wang $\mathrm{R}$ and Brattain MG. The maximal size of protein to diffuse through the nuclear pore is larger than $60 \mathrm{kDa}$. FEBS Lett 581: 3164-3170, 2007.

20. Waxman DJ. Glutathione S-transferases: role in alkylating agent resistance and possible target for modulation chemotherapy-a review. Cancer Res 50: 6449-6454, 1990.

Address correspondence to: Dr. Jin Wang

Department of Pharmacology and Chemical Biology

Baylor College of Medicine Houston, TX 7137986875

E-mail: wangj@bcm.edu

Date of first submission to ARS Central, July 2, 2018; date of final revised submission, October 5, 2018; date of acceptance, October 22, 2018.

$\begin{aligned} & \text { Abbreviations Used } \\ \mathrm{BSO} & =\text { buthionine sulfoximine } \\ \mathrm{GGSH} & =\text { cytosolic glutathione } \\ \mathrm{CMFDA} & =\text { - } \text {-chloromethylfluorescein diacetate } \\ \mathrm{DCM} & =\text { dichloromethane } \\ \mathrm{DEM} & =\text { diethyl maleate } \\ \mathrm{DIPEA} & =\text { N,N-diisopropylethylamine } \\ \mathrm{DMF} & =\text { dimethylformamide } \\ \mathrm{EBSS} & =\text { Earle's balanced salt solution } \\ \mathrm{ER} & =\text { endoplasmic reticulum } \\ \mathrm{FL} & =\text { fluorescence } \\ \mathrm{GPC} & =\text { gel permeation chromatography } \\ \mathrm{GSH} & =\text { glutathione } \\ \mathrm{H}_{2} \mathrm{O}_{2} & =\text { hydrogen peroxide } \\ \mathrm{M} \mathrm{aKK} & =\text { MapK kinase } \\ \mathrm{MCB} & =\text { monochlorobimane } \\ \mathrm{NEL} & =\text { nelfinavir } \\ \mathrm{nGSH} & =\text { nuclear glutathione } \\ \mathrm{NLS} & =\text { nuclear localization sequence } \\ \mathrm{NMM} & =N \text {-methylmaleimide } \\ \mathrm{PBS} & =\text { phosphate-buffered saline } \\ \mathrm{RT} & =\text { RealThiol probe } \\ \mathrm{TFA} & =\text { trifluoroacetic acid } \\ \mathrm{TM} & =\text { tunicamycin } \\ & \end{aligned}$

